\title{
Anti-de Sitter wormhole kink
}

\author{
Pedro F. González-Díaz \\ Centro de Fúsica "Miguel Catalán", Instituto de Matemáticas y Física Fundamental, \\ Consejo Superior de Investigaciones Científicas, Serrano 121, 28006 Madrid (SPAIN)
}

(May 19, 1997)

\begin{abstract}
The metric describing a given finite sector of a four-dimensional asymptotically anti-de Sitter wormhole can be transformed into the metric of the time constant sections of a Tangherlini black hole in a five-dimensional anti-de Sitter spacetime when one allows light cones to tip over on the hypersurfaces according to the conservation laws of an one-kink. The resulting kinked metric can be maximally extended, giving then rise to an instantonic structure on the euclidean continuation of both the Tangherlini time and the radial coordinate. In the semiclassical regime, this kink is related to the existence of closed timelike curves.
\end{abstract}

\section{INTRODUCTION}

The choice of the boundary conditions for the quantum state of the universe should be carried out on just the two natural possibilities which exist for positive definite metrics; i.e.: compact metrics or noncompact metrics which are asymptotic to metrics of maximal symmetry. Choosing either only compact metrics to avoid ${ }^{1}$ any boundary for the quantum state of the universe, or noncompact metrics which are disconnected, consist of a compact part with physical boundary at a given hypersurface and an asymptotically euclidean or anti-de Sitter part without any inner boundary, and dominate on any noncompact connected metric ${ }^{2,3}$, does not make much difference for the resulting state amplitude. For practical purposes, instead of compact metrics ${ }^{1}$, one could likewise take noncompact disconnected metrics for the quantum state of the universe. Therefore, metrics which are asymptotically euclidean or anti-de Sitter may also contribute the path integral describing the state of the universe. Clearly, when such metrics are endowed with a given nonzero boundary which behaves as a microscopic bridge between two asymptotic, large regions, these metrics would represent contributions from wormholes to the quantum state of the universe.

Wormholes whose maximally-symmetric asymptotic metrics are flat space have already been extensively considered ${ }^{4}$. They come about as solutions of the euclidean Einstein equations for gravity coupled to special kinds of matter fields ${ }^{5}$. Contributing connections bridging maximally-symmetric anti-de Sitter asymptotic metrics were first studied in Refs. 6 and 7 for the case of a massless, scalar field which conformally couples to gravity.

Wormhole kinks ${ }^{8}$ can be regarded as topologically admissible generalizations of euclidean wormholes, allowing for a connection between topology changes and black holes ${ }^{9}$. Whereas asymptotically euclidean wormholes and their kinky extensions ${ }^{8}$ have also been studied in terms of spherically symmetric coordinates in spacetimes that show an apparent singularity at the throat radius ${ }^{9}$, the corresponding spherically symmetric representation and kinky extension of wormholes which are asymptotically anti-de Sitter have not hitherto been considered. Since these wormholes have vanishing action (as it can be seen ${ }^{7}$ by addition of suitable surface terms and gauge fixing) one should expect that they would also naturally contribute the probability amplitude for the universe.

The present work aims at bringing such a study to completion by regarding the wormhole metric as a constanttime section of a five-dimensional black hole in anti-de Sitter space, and discussing the possible implications that the existence of asymptotically anti-de Sitter wormhole kinks may have in the presence of closed timelike curves.

Let us first briefly review the general topological concept of a kink and its associated topological charge. Let $\left(\mathbf{M}, g_{a b}\right)$ be a given D-dimensional spacetime, with $g_{a b}$ a Lorentz metric on it. One can always regard $g_{a b}$ as a map from any connected D-1 submanifold $\Sigma \subset \mathbf{M}$ into a set of timelike directions in $\mathbf{M}^{10}$. Metric homotopy can then be classified by the degree of this map. This is seen by introducing a unit line field $\{n,-n\}$, normal to $\Sigma$, and a global framing $u_{i}: i=1,2, \ldots, \mathrm{D}-1$, of $\Sigma$. A timelike vector $\mathbf{v}$ can then be written in terms of the resulting tetrad framing $\left(n, u_{i}\right)$ as $v=v^{0} n+v^{i} u_{i}$, such that $\sum_{i}^{D-1}\left(v^{i}\right)^{2}=1$. Restricting to time orientable manifolds $\mathbf{M}, \mathbf{v}$ then determines a map

$$
K: \Sigma \rightarrow S^{D-1}
$$

by assigning to each point of $\Sigma$ the direction that $\mathbf{v}$ points to at that point. This mapping allows a general definition of kink and kink number. Respect to hypersurface $\Sigma$, the kink number (or topological charge) of the Lorentz metric $g_{a b}$ is defined by ${ }^{10}$

$$
\operatorname{kink}\left(\Sigma ; g_{a b}\right)=\operatorname{deg}(K)
$$


so this topological charge measures ${ }^{11}$ how many times the light cones rotate all the way around as one moves along $\Sigma$.

In the case of an asymptotically flat spacetime the pair $(\Sigma, g)$ will describe an asymptotically flat kink if kink $(\Sigma ; g) \neq$ 0 . All of the topological charge of the kink in the metric $g$ is in this case confined to some finite compact region ${ }^{11}$. Outside that region all hypersurfaces $\Sigma$ are everywhere spacelike. Topology changes, such as handles or wormholes, can occur in the compact region supporting the kink, but not outside it. All topologies are actually allowed to happen in such a region. Therefore, in the case of spherically symmetric kinks, the supporting region should be viewed as an essentially quantum-spacetime construct. This is the view we shall assume throughout this paper.

Imposing to the wormhole space the invariance properties of an one-kink would induce a change in the geometrical structure of the wormhole that resulted in a bridge with smaller cross-sectional area at the neck ${ }^{9}$. When visualized as a constant-time section of a five-dimensional black hole in anti-de Sitter space, one would moreover expect the wormhole metric to correspond to some four-dimensional Einstein-Rosen bridge ${ }^{12}$ in anti-de Sitter space. This space has two problems. First, on the maximal analytical extension of its metric, one of the two wormhole halves must necessarily be described in the nonphysical exterior region ${ }^{13}$, and secondly, this wormhole should inexorably pinch off at the neck, just as it happens in the three-dimensional Einstein-Rosen bridge ${ }^{12,13}$. These difficulties would no longer be expected to occur in the corresponding kinked wormhole. Since the kink number must be conserved, the wormhole ought to be described in two distinct coordinate patches only identified at a given common surface inside the compact region supporting the kink, with a black hole living in each patch. This identification would occur both on the physical and nonphysical regions of the maximal analytical extension, separately, and would represent a bridge which could not pinch off.

The paper is organized as follows. In Sec. II we deal with the metric of asymptotically anti-de Sitter wormholes in terms of spherically symmetric coordinates and avoid its singularity at the neck by a convenient coordinate transformation. Sec. III generalizes this metric to the case where there exists an one-kink. The geodesic incompleteness of the resulting metric is removed by Kruskal extension in Sec. IV and in Sec. V we analytically continue the Kruskal kink metric into the euclidean regime, discussing the instantons that represent formation of black-hole kinks in anti-de Sitter spacetime. We consider the thermal emission of these black holes and the related possibility that the kink be linked to the formation of closed timelike curves in Sec. VI. Finally, we summarize and conclude in Sec. VII.

\section{SPHERICALLY-SYMMETRIC WORMHOLES IN ANTI-DE SITTER SPACE}

Wormholes in asymptotically anti-de Sitter spacetime were first considered in ${ }^{6}$ and dealt with in greater detail in $^{7}$. They correspond to solutions of the euclidean Einstein equations for Hilbert-Einstein gravity with a negative cosmological constant, conformally coupled to a massless scalar field. The semiclassical geometry of these wormholes is described by the isotropic and homogeneous metric

$$
d s^{2}=a(\eta)^{2}\left(d \eta^{2}+d \Omega_{3}^{2}\right)
$$

in which $d \Omega_{3}^{2}$ is the metric on the unit three-sphere, the scale factor $a(\eta)$ is given by

$$
a(\eta)=\left(\frac{b-1}{2 \Lambda}\right)^{\frac{1}{2}} n c\left(b^{\frac{1}{2}} \eta\right)
$$

with $b=\left(1+4 \Lambda M^{2}\right)^{\frac{1}{2}}, \Lambda$ is the absolute value of three times the cosmological constant, $M$ an integration constant, $n c$ the Jacobian elliptic function, and $\eta$ the conformal time $\eta=\int \frac{d \tau}{a}$, where $\tau$ is the Robertson-Walker time.

We note that metric (2.1), (2.2) can also be written in the static, spherically-symmetric form

$$
d s^{2}=\left(1-\frac{M^{2}}{r^{2}}+\Lambda r^{2}\right)^{-1} d r^{2}+r^{2} d \Omega_{3}^{2},
$$

where $M$ can be taken to be the same mass constant as that appearing in the definition of parameter $b$ above, and $\Lambda$ is renormalized so that it becomes also the same as the cosmological constant in $b$.

While it is rather trivial to see that the Robertson-Walker metric given by (2.1) and (2.2) describes the space of a wormhole with a neck at $a(\eta=0)$, it is not so obvious to show that the spherically symmetric metric (2.3) describes a wormhole. In particular, inspection of (2.3) tells us that there is a singularity at $r=\left(\frac{b-1}{2 \Lambda}\right)^{\frac{1}{2}}$ and, therefore, metric (2.3) is geodesically incomplete. In what follows, we shall extend this metric so that it, in fact, becomes regular, with a neck replacing the singularity. 
The singularity at $r=\left(\frac{b-1}{2 \Lambda}\right)^{\frac{1}{2}}$ corresponds to an apparent coordinate singularity, rather than a true curvature singularity. It can be avoided by introducing a new coordinate from the definition

$$
\frac{d r}{\left(1-\frac{M^{2}}{r^{2}}+\Lambda r^{2}\right)^{\frac{1}{2}}}=r d[\ln u(r)]
$$

i.e:

$$
\ln \left(\frac{u(r)}{\mu}\right)=\frac{2 i}{\sqrt{b \Lambda}} F\left[\arcsin \left(\frac{1-\sqrt{\frac{2 \Lambda}{b-1}} r}{2}\right), \sqrt{b+1}\right],
$$

in which $\mu$ is an arbitrary integration constant and $F$ is the elliptic integral of the first kind ${ }^{15}$.

From (2.5) we finally obtain the coordinate transformation

$$
r=\sqrt{\frac{2(b-1)}{\Lambda}}\left(n c\left[\frac{\sqrt{b \Lambda}}{2} \ln \left(\frac{u}{\mu}\right)\right]-\frac{1}{2}\right) .
$$

Using (2.6) we transform (2.1) and (2.2) into the asymptotically anti-de Sitter wormhole metric

$$
d s^{2}=\left(\frac{2(b-1)}{\Lambda}\right)\left(n c\left[\frac{\sqrt{b \Lambda}}{2} \ln \left(\frac{u}{\mu}\right)\right]-\frac{1}{2}\right)^{2}\left(\frac{d u^{2}}{u^{2}}+d \Omega_{3}^{2}\right),
$$

which is everywhere regular and shows a neck at the arbitrary scale $u=\mu$, i.e.: at $r=\sqrt{\frac{b-1}{2 \Lambda}}$, as for metric defined by (2.1) and (2.2). The metric (2.7) gives the geometry of a regular spherically-symmetric wormhole in an asymptotically anti-de Sitter spacetime.

\section{THE TANGHERLINI-ANTI-DE SITTER KINK}

A worth-exploiting feature of metric (2.3) is that it corresponds to the metric of a five-dimensional Tangherlini black hole in anti-de Sitter space

$$
d s^{2}=-\left(1-\frac{M^{2}}{r^{2}}+\Lambda r^{2}\right) d T^{2}+\left(1-\frac{M^{2}}{r^{2}}+\Lambda r^{2}\right)^{-1} d r^{2}+r^{2} d \Omega_{3}^{2}
$$

at a surface with constant time $T=t_{0}$. The relation between (2.3) and (3.1) can be viewed within the context of the kink concept ${ }^{8}$ in the following way: the kink extension of metric (3.1) would also be the kink extension of metric (2.3), with $T=t_{0}$, corresponding to all possible light cone orientations which are compatible with the existence of one kink, starting with a future-directed light cone orientation where the kinked metric should reduce to (2.3) [9]. Thus, we will consider metric (2.3) to correspond to a given fixed value of time $T$ in the kink extension of the Tangherlini anti-de Sitter metric, or in other words, we wish to find an isometric embedding of the four-dimensional wormhole described by metric (2.3), as removed from (3.1), in a space which is the kinked extension of (3.1).

We shall take for the metric that describes a three-spherically symmetric general kink ${ }^{9}$

$$
d s^{2}=-\cos 2 \alpha\left(d t^{2}-d r^{2}\right)-2 \sin 2 \alpha d t d r+r^{2} d \Omega_{3}^{2},
$$

where $\alpha$ is the angle of tilt for the light cones. An one-kink is ensured to exist if $\alpha$ is allowed to monotonously increase from 0 to $\pi$, starting with $\alpha(0)=0$. Then, it can be checked that metric (3.2) converts into (2.3) if we use the substitution

$$
\sin \alpha=\frac{1}{\sqrt{2}}\left(\frac{M^{2}}{r^{2}}-\Lambda r^{2}\right)^{\frac{1}{2}}
$$

with $0 \leq \alpha \leq \pi$, and introduce the change of time variable $t+g(r)=t_{0}$, where $t_{0}$ is the arbitrary constant time labeling the surface on which metric (2.3) is defined from (3.1), with the function $g(r)$ chosen such that $\frac{d g}{d r}=\tan 2 \alpha$. 
For future-directed orientation of light cones, $\alpha=0$, time $t$ becomes also constant and, in fact, metric (3.2) reduces to $(2.3)$, as it was pointed out at the beginning of this section.

Since $\sin \alpha$ cannot exceed unity, it follows that

$$
r_{\max }=\left(\frac{M}{\sqrt{\Lambda}}\right)^{\frac{1}{2}} \geq r \geq r_{\min }=\left(\frac{a-1}{\Lambda}\right)^{\frac{1}{2}},
$$

with $a=\left(1+\Lambda M^{2}\right)^{\frac{1}{2}}$, so that $\alpha$ varies only from 0 to $\frac{\pi}{2}$. It is worth noticing that the presence of a negative cosmological term in the metric makes the asymptotic region $r \rightarrow \infty$ to vanish, leaving a finite maximum surface at $r=r_{\max }$, so that in this case the kink turns out to be a gravitational topological defect with finite volume.

A complete one-kink with finite volume can only be achieved if we add a second coordinate patch in order to describe the other half of the $\alpha$-interval from $\frac{\pi}{2}$ to $\pi$. This extension requires introducing a new time coordinate $\bar{t}=t+h(r)$, where

$$
h=\int d r\left(\frac{d g}{d r}-\frac{k}{\cos 2 \alpha}\right), \quad k= \pm 1,
$$

which transforms (3.2) into the standard kinked metric ${ }^{16}$

$$
d s^{2}=-\cos 2 \alpha d \bar{t}^{2}-2 k d \bar{t} d r+r^{2} d \Omega_{3}^{2} .
$$

For $T=t_{0}$ constant, one can check that time $\bar{t}$ is a function of $r$ alone, and that metric (3.5) converts into metric (2.3) by using $\bar{t}=t_{0}-g(r)+h(r),(3.3)$ and (3.4).

The zeros in the denominator of $\frac{d h}{d r}=(\sin 2 \alpha \mp 1) / \cos 2 \alpha$ occur at the two horizons where $r=r_{h}=\sqrt{\frac{b-1}{2 \Lambda}}$, one per patch. For the first patch, the horizon occurs at $\alpha=\alpha_{h}=\arcsin \left(\frac{b-a^{2}}{\sqrt{2}(b-1)}\right)$ and therefore the upper sign $(k=+1)$ is selected so that $\frac{d h}{d r}$ and $h$ remain well defined and hence the kink is not lost in the transformation from (3.2) to (3.5). The horizon in the second patch takes place at $\alpha=\alpha_{h}+\frac{\pi}{2}$ and therefore the lower sign $(k=-1)$ is selected.

The need for two coordinate patches to describe the complete spacetime of the kink can also be seen by regarding the connection between the wormhole and the kink as an embedding; i.e. we visualize the wormhole metric (2.3) as removed from Tangherlini spacetime and embedded in the kinked spacetime. The embedding surface should then flare outward at $r=r_{\max }$ and inward at $r=r_{\min }$, regularly in both cases. The latter requirement comes from the fact that the maximum cross section of the wormhole is finite and its minimum cross section is nonzero, i.e. the wormhole should avoid pinching off.

The metric (3.2) fails to be the metric of such an embedding; for, though time $t$ is a function of $r$ interpretable as an embedding function such that $d t / d r=-d g / d r=-\tan 2 \alpha$, and hence the rate of embedding

$$
\frac{d^{2} r}{d t^{2}}=\frac{\left(1-\frac{M^{2}}{r^{2}}+\Lambda r^{2}\right)\left(\frac{M^{2}}{r^{3}}+\Lambda r\right)\left[2-\left(\frac{M^{2}}{r^{2}}-\Lambda r^{2}\right)^{2}\right]}{\left(\frac{M^{2}}{r^{2}}-\Lambda r^{2}\right)^{2}\left(1-\frac{M^{2}}{2 r^{2}}+\frac{\Lambda r^{2}}{2}\right)^{2}}
$$

is positive (i.e. the embedding surface flares outward) at $r_{\max }$ and negative (i.e. the embedding surface flares inward) at $r_{\text {min }}, \frac{d^{2} r}{d t^{2}}$ becomes singular at these extreme regions, so that the wormhole pinches off at the second of them.

However, if we choose the standard kinked metric (3.5) and, therefore, we take $\bar{t}=t_{0}-g+h$ as the embedding function, such that $\frac{d \bar{t}}{d r}=-\frac{k}{\cos 2 \alpha}$, then the rate of embedding becomes independent of $k$ and given by

$$
\frac{d^{2} r}{d \bar{t}^{2}}=2\left(1-\frac{M^{2}}{r^{2}}+\Lambda r^{2}\right)\left(\frac{M^{2}}{r^{3}}+\Lambda r\right)
$$

which is positive at $r=r_{\max }$,

$$
\left.\frac{d^{2} r}{d \bar{t}^{2}}\right|_{r_{\max }}=\frac{4 M^{2}}{r_{\max }^{3}},
$$

negative at $r_{\text {min }}$,

$$
\left.\frac{d^{2} r}{d \bar{t}^{2}}\right|_{r_{\text {min }}}=-4 \frac{\left(\frac{M^{2}}{r_{\min }^{2}}-1\right)}{r_{\min }}
$$


and regular everywhere. Thus, the wormhole turns out to be connectible to the complete kinked spacetime with metric (3.5) at both extreme surfaces of the wormhole; that is to say, the wormhole (2.3) can be interpreted as a kinked boundary in the space with metric (3.5), in each of the two coordinate patches.

Although still geodesically-incomplete, metric (3.5) will describe the complete interval of the light-cone configurations running from light cones that point vertically up and out from the finite maximum surface at $\alpha=\pi$, to light cones that point vertically up and into the other maximum surface at $\alpha=\pi$, and this is only possible if two coordinate patches identified on the minimum surface at $\alpha=\frac{\pi}{2}$ are used.

On the other hand, every kink is characterized by a topological charge or kink number ${ }^{9}$. If, as in the case being considered, one has an one-kink, the topological charge can be either positive when the light cones rotate away from external observers (that is a black hole in anti-de Sitter space), or negative when the light cones rotate in the opposite direction for the case of a white hole in anti-de Sitter space. In the latter case, the sign of the crossed metric component $g_{t r}$ in (3.2), or $g_{\bar{t} r}$ in (3.5), is changed in each coordinate patch.

A specific characteristic of the considered kink is that, for the real values of the angle $\alpha$ running from 0 to $\pi$, the resulting kinked spacetime covers the sector of the original kinkless metric going up to $r_{\max }$ only. One still could cover the spacetime up to $r=\infty$ by analytically continuing the tilt angle so that $\alpha \rightarrow \alpha+i \tilde{\alpha}$, allowing it to monotonously run first from $\alpha=0, \tilde{\alpha}=+\infty$, at $r=\infty$, to $\alpha=0, \tilde{\alpha}=0$, at $r_{\max }$, along the first corrdinate patch, then to $\alpha=\pi$, $\tilde{\alpha}=0$, at $r_{\max }$, after crossing to the second patch, and finally to $\alpha=\pi, \tilde{\alpha}=-\infty$, at $r=\infty$, on the second patch. Here, the rotation of the light cones over the hypersurfaces is taken to occur in the complex $\alpha$-plane, and the sectors of the original spacetime beyond $r_{\max }$ are covered by monotonous variation of the purely imaginary parameter $\tilde{\alpha}$.

\section{KRUSKAL EXTENSION}

The standard kinked metric for black or white holes,

$$
d s^{2}=-\cos 2 \alpha d \bar{t}^{2} \mp 2 k d \bar{t} d r+r^{2} d \Omega_{3}^{2},
$$

still contains a geodesic incompleteness at the horizon $r=r_{h}=\left(\frac{b-1}{2 \Lambda}\right)^{\frac{1}{2}}$, in each patch. These apparent singularities can be removed by using the Kruskal technique ${ }^{17}$. In order to achieve the maximally-extended metric, let us introduce the general metric

$$
d s^{2}=-H(U, V) d U d V+r^{2} d \Omega_{3}^{2},
$$

where

$$
\begin{gathered}
H=\left(\frac{b-1}{2 \Lambda}\right)^{\frac{1}{2}} \frac{\cos 2 \alpha}{\beta} \exp \left(-2 k \beta \int_{r_{\max } / r_{\min }}^{r} \frac{d r}{\cos 2 \alpha}\right) \\
U=\mp e^{\beta \bar{t}} \exp \left(2 k \beta \int_{r_{\max } / r_{\min }}^{r} \frac{d r}{\cos 2 \alpha}\right) \\
V=\mp\left(\frac{2 \Lambda}{b-1}\right)^{\frac{1}{2}} \frac{e^{-\beta \bar{t}}}{2 \beta}
\end{gathered}
$$

the constant $\beta$ in (4.2)-(4.4) is an adjustable parameter to be chosen such that the unphysical singularity at $r=r_{h}$ is removed, and the lower integration limit $r_{\max } / r_{\min }$ accounts for the choices $r=r_{\max }$ and $r=r_{\min }$, depending on whether the first or second patch is being considered. Using (3.3), we obtain for (4.2):

$$
\begin{gathered}
H=\frac{\sqrt{\Lambda(b-1)}\left(r^{2}-\frac{b-1}{2 \Lambda}\right)\left(r^{2}+\frac{b+1}{2 \Lambda}\right)}{\sqrt{2} \beta r^{2}} \\
\times \exp \left\{-\frac{\sqrt{2(b+1)} k \beta}{\sqrt{\Lambda} b} \arctan \left[\sqrt{\frac{2 \Lambda}{b+1}} r\right]\right\} \times\left[\frac{\left.\left(\sqrt{\frac{b-1}{2 \Lambda}}+r\right)^{2}\right]^{\sqrt{\frac{b-1}{2 \Lambda}} \frac{k \beta}{b}}}{\frac{b-1}{2 \Lambda}-r^{2}}\right] .
\end{gathered}
$$


Eqn. (4.5) would actually have some constant term coming from the lower integration limit $r_{\max } / r_{\min }$. This term has been omitted because it is canceled by the similar constant term of the Kruskal coordinate $U$ when forming the Kruskal metric.

Geodesic incompleteness is avoided if we choose

$$
\beta=\frac{\sqrt{2 \Lambda} b}{k \sqrt{b-1}}
$$

Hence, we finally obtain for the Kruskal metric

$$
d s^{2}=-\frac{k(b-1)\left(r^{2}+\frac{b-1}{2 \Lambda}\right)\left[\sqrt{\frac{b-1}{2 \Lambda}}+r\right]^{2}}{2 b r^{2}} \times \exp \left[-2 \sqrt{\frac{b+1}{b-1}} \arctan \left(\sqrt{\frac{2 \Lambda}{b+1}} r\right)\right] d U d V+r^{2} d \Omega_{3}^{2},
$$

with

$$
\begin{gathered}
U=\mp e^{b k \sqrt{\frac{2 \Lambda}{b-1}} \bar{t}}\left(\frac{\sqrt{\frac{b-1}{2 \Lambda}}-r}{\sqrt{\frac{b-1}{2 \Lambda}}+r}\right) \times \exp \left[2 \sqrt{\frac{b+1}{b-1}} \arctan \left(\sqrt{\frac{2 \Lambda}{b+1}} r\right)\right] \\
V=\mp \frac{k e^{-b k \sqrt{\frac{2 \Lambda}{b-1}} \bar{t}}}{2 b},
\end{gathered}
$$

where

$$
\bar{t}=\bar{t}_{0}(k)-\frac{k}{b} \sqrt{\frac{b+1}{2 \Lambda}} \arctan \left(\sqrt{\frac{2 \Lambda}{b+1}} r\right)+\frac{k}{2 b} \sqrt{\frac{b-1}{2 \Lambda}} \ln \left(\frac{1+\sqrt{\frac{2 \Lambda}{b-1}} r}{1-\sqrt{\frac{2 \Lambda}{b-1}} r}\right)
$$

in (4.10) the constant coming from the lower integration limit has been absorbed into the constant term $\bar{t}_{0}(k)$ whose value will therefore depend on the coordinate patch we are considering.

By allowing $\alpha$ to be analytically continued to complex values, and the lower integration limit for the first patch to become then infinity, the integration in the expressions for $H$ and $U$ could be performed along the continuous path described at the end of Sec. IV, so obtaining a truly anti-de Sitter asymptotic behaviour for the maximally extended kinked metric. The resulting metric should be compared with the extended asymptotically anti-de Sitter wormhole metric (2.7) which is defined in one coordinate patch only. It can be seen that the form of (4.7) coincides with that of the Kruskal extension of the five-dimensional Tangherlini-anti-de Sitter kink and differs from the kinkless counterpart of this just by the presence in (4.7) of the sign parameter $k$. We also note that in the limit $\Lambda \rightarrow 0,(4.7)$ reduces to the kinked five-dimensional black hole metric.

Continuity of the tilt angle also at $\frac{\pi}{2}$ ensures the identification of the two coordinate patches on the surface $r=r_{\min }$. Such an identification should be done both on the original physical regions and on the new, nonphysical regions created by the Kruskal extension, separately. It gives rise to bridges that connect the maximal surfaces at $r_{\max }$ of the asymptotically anti-de Sitter regions in the two patches. Any constant time $T=\bar{t}_{0}$ sections of the resulting spacetime will then describe four-dimensional wormholes occurring either in the physical or nonphysical regions, whose neck is now at $r=r_{\text {min }}$, rather than $r=\left(\frac{b-1}{2 \Lambda}\right)^{\frac{1}{2}}$, in asymptotic anti-de Sitter space, such as it was announced in the Introduction.

Finally, we can now see why the studied classical kink cannot be linked to the presence of closed timelike curves. At first glance, it might seem that the above discussed identification between the two patches on $r=r_{\min }$ would allow us to choose a null geodesic such that it started in a given largest-surface of the original regions and would somehow "loop back" through the new regions to finally arrive at its starting point. However, one can easily convince oneself that such an itinerary is classically disallowed, since it requires identification of the two patches also on maximum surfaces belonging to a physical and a nonphysical region, respectively (see Fig. 1).

\section{THE INSTANTONS}

Euclidean continuations of the metric that contains one kink must be obtained by using the Wick rotation 


$$
\bar{t} \rightarrow i \bar{\tau}
$$

with $\bar{t}$ as given in (4.10). Because of time redefinition, the instanton structure is richer than in the kinkless case. Thus, it will be shown in what follows that the euclidean continuation (5.1) should give rise to metrics which are positive definite only if we choose either the usual continuation $\bar{t}_{0} \rightarrow i \bar{\tau}_{0}$, for $r \geq r_{h}$ and $k=+1$, or the new kinky continuation $r \rightarrow-i \rho, M \rightarrow-i \mu, \Lambda \rightarrow-\lambda$, for $r<r_{h}$ and $k=-1$, where $r$ becomes timelike and we transform a space coordinate into a time coordinate.

In order to see how this can be, let us consider the new variables ${ }^{18} y+z=U$ and $y-z=V$ in the Kruskal metric (4.7), which then becomes:

$$
d s^{2}=-k G(\Lambda, M, r)\left(d y^{2}-d z^{2}\right)+r^{2} d \Omega_{3}^{2},
$$

with

$$
G(\Lambda, M, r)=\frac{k(b-1)\left(r^{2}+\frac{b-1}{2 \Lambda}\right)\left[\sqrt{\frac{b-1}{2 \Lambda}}+r\right]^{2}}{2 b r^{2}} \times \exp \left[-2 \sqrt{\frac{b+1}{b-1}} \arctan \left(\sqrt{\frac{2 \Lambda}{b+1}} r\right)\right] .
$$

We have furthermore

$$
\begin{gathered}
y^{2}-z^{2}=k J(\Lambda, M, r)\left(\frac{1-\sqrt{\frac{2 \Lambda}{b-1}} r}{1+\sqrt{\frac{2 \Lambda}{b-1}} r}\right), \\
\frac{y+z}{y-z}=2 k b e^{2 b \sqrt{\frac{2 \Lambda}{b-1}} k \bar{t}_{0}},
\end{gathered}
$$

with

$$
J(\Lambda, M, r)=\frac{1}{2 b} \exp \left[2 \sqrt{\frac{b+1}{b-1}} \arctan \left(\sqrt{\frac{2 \Lambda}{b+1}} r\right)\right] .
$$

The singularity at $r=0$ would lie on the surfaces $y^{2}-z^{2}=\frac{k}{2 b}$. This singularity can be avoided by defining either a new coordinate $\zeta=i y$, or a new coordinate $\xi=i z$. For the first choice, the metric takes the form

$$
d s^{2}=k G(\Lambda, M, r)\left(d \zeta^{2}-d z^{2}\right)+r^{2} d \Omega_{3}^{2},
$$

which is positive definite in the patch $k=+1$ and has the signature --++ in the patch $k=-1$. The radial coordinate $r$ is then defined as

$$
\zeta^{2}+z^{2}=k J(\Lambda, M, r)\left(\frac{r-\sqrt{\frac{b-1}{2 \lambda}}}{r+\sqrt{\frac{b-1}{2 \lambda}}}\right) .
$$

On the section on which $\zeta$ and $z$ are both real, $\sqrt{\frac{M}{\sqrt{\Lambda}}} \geq r \geq r_{h} \equiv \sqrt{\frac{b-1}{2 \Lambda}}$ for $k=+1$, and $\sqrt{\frac{a-1}{\Lambda}} \leq r \leq r_{h}$ for $k=-1$. Define the imaginary time by $\bar{t}_{0} \rightarrow i \bar{\tau}_{0}$. This continuation leaves invariant the form of the metric (5.7) and therefore is compatible with the coordinate transformation $\zeta=i y$. Then, from (5.5) we obtain

$$
z-i \zeta=\sqrt{2 b\left(z^{2}+\zeta^{2}\right) k} i e^{i b \sqrt{\frac{2 \Lambda}{b-1}} k \bar{\tau}_{0}}
$$

It follows that for this time continuation, $\bar{\tau}_{0}$ is periodic with a period $2 \pi k / b \sqrt{\frac{2 \Lambda}{b-1}}$. On this nonsingular euclidean section (which would correspond to the usual euclidean section for the patch $k=+1$ ) $\bar{\tau}_{0}$ has the character of an angular coordinate which would rotate, clockwise about the "axis" $r=r_{h}$ in patch $k=+1$, and anti-clockwise about the "axis" $r=0$ in patch $k=-1$.

For the other choice of coordinates, $\xi=i z$, metric (5.2) takes the form:

$$
d s^{2}=-k G(\Lambda, M, r)\left(d y^{2}+d \xi^{2}\right)+r^{2} d \Omega_{3}^{2},
$$


which is definite positive only in patch $k=-1$, and has signature - - + + in patch $k=+1$. In this case, the radial coordinate is defined by

$$
\xi^{2}+y^{2}=k J(\Lambda, M, r)\left(\frac{\sqrt{\frac{b-1}{2 \lambda}}-r}{\sqrt{\frac{b-1}{2 \lambda}}+r}\right) .
$$

On the section on which $y$ and $\xi$ are both real, it follows now $\sqrt{\frac{M}{\sqrt{\Lambda}}} \geq r \geq r_{h} \equiv \sqrt{\frac{b-1}{2 \Lambda}}$ for $k=-1$, and $\sqrt{\frac{a-1}{\Lambda}} \leq r \leq r_{h}$ for $k=+1$. In this case, we can introduce the transformation $r=-i \rho, M=-i \mu, \Lambda=-\lambda$, keeping $\bar{t}_{0}$ real. In order for this transformation to be compatible with the coordinate redefinition $\xi=i z$, it should leave metric (5.10) formally unchanged. This is accomplished by also continuing the line element itseft, $d s=-i d \sigma$, in accordance with the introduction of the "tachyonic" mass $M=-i \mu$. Thus, we have

$$
y-i \xi=\sqrt{2 b\left(y^{2}+\xi^{2}\right) k} e^{i b \sqrt{\frac{2 \Lambda}{b-1}} k \bar{\tau}_{0}} .
$$

For this kind of time continuation it follows from (5.12) that it is now the lorentzian time $\bar{t}_{0}$ which is periodic with period $2 \pi k / b \sqrt{\frac{2 \lambda}{b-1}}$. On this new nonsingular section $\bar{t}_{0}$ would have the character of an angular coordinate which would rotate about the "axis" $\rho=0$, clockwise in patch $k=+1$ and anti-clockwise in patch $k=-1$.

In the patch $k=+1$, the euclidean continuation (5.1) contains both the continuation for time $\bar{t}_{0}=i \bar{\tau}_{0}$, where the apparent singularity at $r=r_{h}$ is like the irrelevant singularity at the origin of polar coordinates, provided that $\bar{\tau}_{0} / \sqrt{\frac{b-1}{2 b^{2} \Lambda}}$ is regarded as an angular variable and is identified with a period of $2 \pi$, and a new continuation $r=-i \rho$, that also implies the "tachyonic" continuations $M=-i \mu, \Lambda=-\lambda, d s=-i d \sigma$, where the singularity at $\rho=0$ becomes again harmless if $\bar{t}_{0} / \sqrt{\frac{b-1}{2 b^{2} \Lambda}}$ is regarded as an angular variable with period $2 \pi$. For the patch $k=-1$, the continuation (5.1) leads to the same instantonic sections as for the patch $k=+1$, but now $\bar{t}_{0}=i \bar{\tau}_{0}$ corresponds to the section inside the horizon $r=r_{h}$, up to $r=r_{\min }$, and $r=-i \rho, M=-i \mu, \Lambda=-\lambda, d s=-i d \sigma$ define the section outside the horizon $\rho=\rho_{h}$, up to $\rho=\rho_{\max }$, with $\bar{\tau}_{0}$ and $\bar{t}_{0}$ rotating, respectively, about $r=0$ and $\rho_{h}$, anti-clockwise in both cases.

On any $\left(\bar{\tau}_{0}, r\right)$ plane in the coordinate patch $k=+1$ we can define ${ }^{18}$ the transition amplitude $\left\langle\bar{\tau}_{02} \mid \bar{\tau}_{01}\right\rangle$ from the surface $\bar{\tau}_{01}$ to the surface $\bar{\tau}_{02}$ as given by the action of the first instanton. This action can be expressed as the surface area of the circular sector limited by times $\bar{\tau}_{01}$ and $\bar{\tau}_{02}$ of a circle centered at $r=r_{h}$, with radius $r_{\max }$. Similarly, on the plane $\left(\bar{t}_{0}, \rho\right)$ in patch $k=-1$, the transition amplitude $\left\langle\bar{t}_{02} \mid \bar{t}_{01}\right\rangle$ from the surface $\bar{t}_{01}$ to the surface $\bar{t}_{02}$ is dominated by the action of the second instanton and is given by the surface area of the sector limited by $\bar{t}_{01}$ and $\bar{t}_{02}$, from $\rho=\rho_{\text {min }}$ to $\rho=\rho_{h}$, on a circle centered at $\rho=0$. An asymptotic observer in the patch would interpret these transition amplitudes as providing the probability of the occurrence in the vacuum state of, respectively, a five-dimensional black hole with mass $M$ and a five-dimensional white hole with mass $\mu$. In the coordinate patch $k=-1$, the observer would obtain the same interpretation, but for a black hole with mass $M$ or a white hole with mass $\mu$.

\section{THERMAL EMISSION AND CLOSED TIMELIKE CURVES}

At the end of Sec. IV, we argued that the existence of closed timelike curves (CTCs) in the spacetime under consideration is classically disallowed because we cannot identify maximum curves belonging to different coordinate patches. However, in what follows we shall show how such CTCs can still be present in the semiclassical treatment.

The semiclassical regime for kinked spacetimes with event horizons can simply be achieved by considering ${ }^{9}$ the mathematical implications imposed by the fact that time $\bar{t}$ enters the Kruskal coordinates $U, V$ in the form of the dimensionless exponent $\sqrt{\frac{2 \Lambda b^{2}}{b-1}} k \bar{t}$. Hence, the argument of the logarithm in (4.10) becomes square rooted and, therefore, the expression for the time $\bar{t}$ entering the definition of coordinates $U, V$ should be generalized to ${ }^{9}$ :

$$
\bar{t} \rightarrow \bar{t}_{g}=\bar{t}+i \pi \kappa(1-\kappa) k \sqrt{\frac{b-1}{8 b^{2} \Lambda}}
$$

where $\bar{t}$ is given by (4.10) and $\kappa= \pm 1$. For $\kappa=+1, \bar{t}_{g}=\bar{t}$, and for $\kappa=-1$, the points $\left(\bar{t}-i k \pi \sqrt{\frac{b-1}{2 b^{2} \Lambda}}, r, \Omega_{3}\right)$ in each patch are actually the points in the same patch obtained by refletion in the bifurcation point $U, V=0$, keeping the Kruskal metric real and unchanged. 
We note that one can still recover the standard kink metric (3.5) from the general metric (4.1) if we redefine the Kruskal coordinates as follows:

$$
\begin{gathered}
U=\tilde{U}= \pm 2 b \kappa J(\Lambda, M, r) e^{k \sqrt{\frac{2 b^{2} \Lambda}{b-1}} \bar{t}_{c}}\left[\frac{\sqrt{\frac{b-1}{2 \Lambda}}-r}{\sqrt{\frac{b-1}{2 \Lambda}}+r}\right] \\
V=\tilde{V}= \pm k \kappa e^{-k \sqrt{\frac{2 b^{2} \Lambda}{b-1}} \bar{t}_{c}},
\end{gathered}
$$

where

$$
\bar{t}_{c}=\bar{t}+i \pi \kappa k \sqrt{\frac{b-1}{2 b^{2} \Lambda}}
$$

with $\bar{t}=-\operatorname{Re}\left(\int \tan 2 \alpha d r\right)$.

This choice leaves the expressions for $U V=\tilde{U} \tilde{V}, F, r$ and the Kruskal metric real and unchanged. For $\kappa=-1$, Eqns. (4.8) and (4.9) become the sign-reversed of (6.2) and (6.3), respectively; i.e.: the points $\left(\bar{t}-i k \pi \sqrt{\frac{b-1}{2 b^{2} \Lambda}}, r, \Omega_{3}\right)$ on the Kruskal diagrams of the two coordinate patches are the points in the new regions for $r_{h} \leq r \leq r_{\max }$ on the same diagrams, obtained by reflecting in the origins of the respective $U, V$ planes, preserving the Kruskal metric real and unchanged. This leads to identification of hyperbolae in the new regions for $r_{h} \leq r \leq r_{\max }$ with hyperbolae in the original regions for the same values of $r$. We note that the existence of such identifications in turn amounts to both the kind of periodicity required by Hawking thermal radiation ${ }^{19}$ in each patch, and the existence of CTCs.

Using the procedure of Hartle and Hawking ${ }^{19}$ to study the evolution of a scalar field along null geodesics that start at $r_{\max }$ on the physical regions of the Kruskal diagrams for $\kappa=-1$, it becomes now quite natural (in particular, physical time need not be made complex) to obtain that an observer in the exterior original region of the patch $k=+1(-1)$ will measure an isotropic thermal bath of background radiation with positive(negative) energy, at the temperature

$$
T=\frac{2 \pi}{\sqrt{\frac{2 b^{2} \Lambda}{b-1}}} .
$$

This temperature exactly corresponds to the period of the periodic time $\bar{\tau}_{0}$ that we obtained for the instantons in Sec. V.

On the other hand, since in the semiclassical description, we can identify maximum surfaces of the physical regions with those of the nonphysical regions in each coordinate patch separately, we recover allowance for the null geodesics that start at the maximum surface in region $\mathrm{I}_{+}$of patch $k=+1$ (Fig. 1) to continue propagating on patch $k=-1$, after the maximum surface of region III - , first through region $\mathrm{II}_{-}$and then through $\mathrm{IV}_{-}$, up to the minimum surface of the latter new region. Since this surface can be identified with the similar surface in region $\mathrm{III}_{+}$of patch $k=+1$, the considered null geodesics can thereafter propagate into the region $\mathrm{IV}_{+}$and, again by quantum identification of maximum surfaces, come back to their starting points on the maximum surface of region $\mathrm{I}_{+}$, in patch $k=+1$.

Hence, null geodesics starting from large original regions can still "loop back" to arrive to their starting points, so completing a CTC, provided such a CTC is involved at a thermal radiation process preventing any information to flow from or to the CTC. Gibbons and Hawking ${ }^{10}$ have recently suggested the possibility of linking the presence of kinks to the presence of CTCs. However, Chamblin and Penrose ${ }^{20}$ have subsequently shown that CTCs are not a classical requirement for kinked spacetimes. Our result therefore fits in with these works, since we obtain that CTCs are linked to kinks only when matter traveling through these spacetimes is considered quantum-mechanically.

\section{CONCLUSION}

In this work, we have constructed the spherically-symmetric spacetime metric describing the geometry of wormholes which are asymptotically anti-de Sitter, generalizing this metric to the case where the light cones are allowed to tip over on hypersurfaces. By using the kink geometry, a relation between four-dimensional wormholes and five-dimensional Tangherlini black holes has been established in the anti-de Sitter background.

We have seen that at least two coordinate patches are needed for a complete description of the kink. Since the presence of an event horizon makes the kink metric geodesically incomplete, we have maximally extended this metric 
by resorting to the Kruskal technique. On each spacelike slice of the kinked Tangherlini anti-de Sitter space, the kinked wormhole results as a bridge between two black holes, one in each patch, at a surface inside the horizon. The space of this wormhole can be completely described on the physical regions of the maximal analytical extension. The extended kink metric is used to study the instantons that can be associated with the kinked Tangherlini-anti-de Sitter black holes. Euclidean continuation of the metrical time of this kink implies both a continuation of the time entering the Tangherlini metric and a continuation of the radial coordinate, removing singularity at the origin.

It has been also shown that the black hole in one coordinate patch emits a thermal bath formed by the antiparticles to the particles that make up the radiation emitted by the hole described in the other patch. Finally, we have also seen that, although this spacetime kink does not imply existence of closed timelike curves classically, when the involved black holes emit Hawking radiation, the kink is always endowed with the formation of such curves.

\section{ACKNOWLEDGMENTS}

This research was supported by DGICYT under research project N PB94-0107, and by MEC Spanish German Joint Action N 161.B. The author thanks U. Kasper for hospitality at Potsdam University where part of this work was done.

[1] S.W. Hawking, in Astrophysical Cosmology, Pontificae Scientiarum Scripta Varia, Vatican City, eds. H.A. Bruck, G.V. Coyne and M.S. Longair, 1982.

[2] J.B. Hartle and S.W. hawking, Phys. Rev. D28, 2960 (1983).

[3] S.W. Hawking, Nucl. Phys. B239, 257 (1983).

[4] S.W. Hawking, Phys. Rev. D37, 904 (1988); S.W. Hawking and D.N. Page, Phys. Rev. D42, 2655 (1990); S. Coleman, Nucl. Phys. B307, 867 (1988); P.F. González-Díaz, Phys. Rev. D42, 3983 (1990); D45, 499 (1992); Nucl. Phys. B351, 767 (1991).

[5] J.J. Halliwell and R. Laflamme, Class. Quant. Grav. 6, 1839 (1989); A. Zhuk, Phys. Rev. D45, 1192 (1992); P.F. GonzálezDíaz, Phys. Rev. D40, 4184 (1989). Grav. Cosm. 2, 45 (1996).

[6] P.F. González-Díaz, Elliptic and Circular Wormholes, IMAFF-RC-02-93; gr-qc/9306031.

[7] C. Barceló, L.J. Garay, P.F. González-Díaz and G.A. Mena Marugán, Phys. Rev. D53, 3162 (1996).

[8] D. Finkelstein and C.W. Misner, Ann. Phys. (N.Y.) 6, 230 (1959); D. Finkelstein, in Directions in General Relativity I, eds. B.L. Hu, M.P. Ryan Jr. and C.V. Vishveshwara (Cambridge Univ. Press, Cambridge, UK, 1993).

[9] P.F. González-Díaz, Phys. Rev. 51D, 7144 (1995); Grav. Cosm. 2, 621 (1996).

[10] G.W. Gibbons and S.W. Hawking, Phys. Rev. Lett. 69, 1719 (1992).

[11] A. Chamblin, Kinks and Singularities, DAMTP preprint R95/44.

[12] A. Einstein and N. Rosen, Phys. Rev. 48, 73 (1935).

[13] R.M. Wald, General Relativity (The University of Chicago Press, Chicago, USA, 1984).

[14] C.W. Misner, K.S. Thorne and J.A. Wheeler, Gravitation (Freeman, New York, USA, 1973).

[15] M. Abramowitz and I. Stegun, Handbook of Mathematical Functions (Dover, New York, 1972).

[16] D. Finkelstein and G. McCollum, J. Math. Phys. 16, 2250 (1975).

[17] M.D. Kruskal, Phys. Rev. 119, 1743 (1960).

[18] G.W. Gibbons and S.W. Hawking, Phys. Rev. D15, 2752 (1977).

[19] J.B. Hartle and S.W. Hawking, Phys. Rev. D14, 2188 (1976).

[20] A. Chamblin and R. Penrose, Twistor Newsletter 34, 13 (1992).

\section{Figure Legend}

Fig. 1.- Kruskal diagrams for the two coordinate patches of the one-kink anti-de Sitter wormhole spacetime. The trajectories for classical geodesics are shown as continuous lines, and the quantum identifications of maximum surfaces and the subsequent geodesic trajectories as dashed lines. 